\title{
EL TALLER DE MECÁNICA AUTOMOTRIZ CHACHI CAR Y LOS INDICADORES DE GESTIÓN
}

\section{THE CHACHI CAR AUTO REPAIR SHOP AND MANAGEMENT INDICATORS}

\author{
José Carlos Véliz ${ }^{1}$
}

\author{
Palabras clave: \\ Comunicación \\ Indicadores de \\ Gestión \\ Liderazgo
}

\begin{abstract}
Resumen
El presente caso nos muestra la situación de la empresa Automotriz Chachi Car la cual con ayuda de los hermanos de Juan Carlos buscan ser los referentes y seguir agregando valor en el mercado automotriz. $\mathrm{Al}$ inicio de la empresa buscaban solamente incrementar sus ventas, pero después analizan que deben de conocer algunos indicadores de gestión como financieros, de operaciones, entre otros y buscan convencer a Juan Carlos la importancia de los indicadores de gestión y su posterior aplicación Códigos JEL: M10
\end{abstract}

\begin{abstract}
The present case shows us the situation of the company Automotriz Chachi Car, which with the help of Juan Carlos' brothers seeks to be the reference point and continue adding value in the automotive market. At the beginning of the company, they were only looking to increase their sales, but later they analyzed that they should know some management indicators such as financial and operations indicators, among others, and tried to convince Juan Carlos of the importance of management indicators and their subsequent application.
\end{abstract}

\footnotetext{
${ }^{1}$ Pontificia Universidad Católica del Perú, Graduate Business School, CENTRUM Católica, Lima, (Perú). E-mail: jcveliz@pucp.edu.pe
} 


\section{INTRODUCCIÓN}

Juan Carlos el mayor de tres hermanos, fundó la empresa llamada Taller de Mecánica Automotriz Chachi Car con mucha esperanza y anhelo de ser un negocio próspero y exitoso, pues tenía como meta ser una empresa referente en el mercado y ganar dinero. Recientemente, había vendido un negocio dedicado a la venta de muebles porque ya había recuperado su inversión. Juan Carlos, siempre deseaba tener un taller de mecánica desde muy niño pues era aficionado a los autos, pero siempre tenía algunas dudas de cómo manejar este tipo de negocio y tenía la esperanza de que sus hermanos que conocían de este tipo de empresa y habían estudiado mecánica automotriz lo puedan ayudar a la administración de este emprendimiento. Después de muchas caminatas y ver diferentes locales decide comprar para instalar su negocio un pequeño garaje en la avenida los Surquillanos. Este garaje tenía una buena ubicación y estaba cerca de una estación de gasolina, además de un lugar de venta de carros de segunda mano.

\section{LOS INICIOS DE LA EMPRESA}

Al día siguiente en compañía de sus hermanos Carlos y Francisco expertos en mecánica y con estudios técnicos en administración en el Instituto TECHMULT deciden juntos ver el local y le comentan a Juan Carlos: "Vamos hacer un gran equipo juntos y nos gusta este local para emprender este negocio, te vamos ayudar hermano, no te preocupes confía en nosotros, somos familia, vamos que se puede". Carlos, quien también había estudiado una carrera técnica en contabilidad le dice a su hermano mayor: "esto es lo mío, los números no te preocupes, además tenemos un asistente que nos ayudará”.

Juan Carlos inaugura la empresa al mes siguiente con auspiciadores, como por ejemplo aceites XCON, y lubricantes PRISSTON, y a los pocos días empiezan a llegar muchos clientes para dar mantenimiento a sus carros, revisiones técnicas y a Juan Carlos se le veía contento y muy feliz, pero al final del año veía que el negocio no estaba bien pues no veía muchos carros en el taller. Hay que considerar que cuando empezó a funcionar la empresa contrato a más de 15 mecánicos y además los puso a todos en planilla con los beneficios sociales correspondientes.

Los hermanos Carlos y Francisco estaban un poco preocupados, pues tampoco veían mucha afluencia de clientes, pero confiaban en su experiencia en que las cosas mejorarían y tenían en mente realizar una campaña de marketing y ventas, contratar más personal de ventas, e invertir en publicidad para tener mayores clientes.

\section{LOS PRIMEROS PROBLEMAS}

Juan Carlos cuando ya está por terminar el primer año de operación de la empresa, decide analizar las ventas junto con sus hermanos y ve estos números: 35000, 54500, 25000, 15000 dólares por ingresos de ventas en el primer, segundo y tercer trimestre. Esto es preocupante exclamo Juan Carlos, veo que no han desagregado la información, no puedo analizar bien los números y solamente veo por trimestres los ingresos por ventas, además hay una alta rotación de personal pues cada dos mes veo que salen 5 mecánicos y reponemos otros 5 .

Francisco se une al análisis, quien estaba tomando su café y había estudiado algo de administración y comenta: "Las ventas están bajando porque no somos competitivos en el mercado, nuestros mejores empleados se van a la competencia, esta empresa es la "escuelita" le enseñamos el A,B,C de la mecánica se van por mas sueldo, mejores condiciones laborales y estamos invirtiendo mucho en ellos algo está mal hermanos, además no invertimos en publicidad y esto repercute en las ventas".

Juan Carlos quien ve que los números no les favorece decide escuchar sus hermanos e invertir en marketing publicidad, contratar más personal y se toma la cabeza y piensa: "mis hermanos han estudiado, ellos saben este tema de la administración".

$\mathrm{Al}$ año siguiente después de la fiesta de año nuevo, la cual fue realizada en un gran hotel de la ciudad en compañía de toda la familia y los amigos, decide que ese año era su año, y decide invertir más de USD\$ 100,000 dólares en 
marketing. Carlos y Francisco celebraron esa decisión y comentaron: "Grande Juan Carlos eres lo máximo que buena idea, hay que seguir invirtiendo en marketing eso nos va ayudar y nos llevara a la salvación de la empresa".

A mediado de año, Juan Carlos decide revisar las ventas de la empresa, pero sus hermanos le dicen, "Juan Carlos nos parece que debemos de ver si estamos cumpliendo las metas no solamente las ventas eso es un indicador, pero los otros indicadores también son importantes".

\section{LAS POSIBLES SOLUCIONES}

Juan Carlos, que primera vez escucha la palabra indicador, le pregunta a Carlos y Francisco: "Hermanos que es eso de indicadores, son importantes o no, otras empresas lo usan sí o no". Carlos y Francisco se miran y le comentan, "un indicador nos va ayudar en el análisis si estamos haciendo bien nuestro trabajo, compararnos con la competencia, seguir mejorando, y realizar los ajustes si no llegamos a las metas y replantear las acciones que debemos de hacer, es sumamente importante implementarlo y no guiarnos de las ventas".

En ese momento Juan Carlos piensa que es una idea excelente pero no sabe por dónde empezar a realizarlos, sus hermanos deciden mostrarle en su pizarra acrílica que existen otros indicadores a parte del nivel de ventas, pues hay de desempeño financieros, recursos humanos, de marketing. Juan Carlos les comenta que muchos indicadores para un pequeño negocio no son factibles, que solo hay que enfocarnos en las ventas y eso nos dirá que hacemos bien o no las cosas.

Los hermanos Carlos y Francisco conversan nuevamente con Juan Carlos en la oficina y le explican en su pizarra acrílica y le dicen: "Juan Carlos hay que primero analizar cuál es la visión, misión, valores, objetivos que tenemos, analizar las perspectivas financieras, clientes, aprendizaje y crecimiento, procesos".

Juan Carlos decide escucharlos, pero sigue creyendo que solamente las ventas son importantes, Carlos y Francisco deciden preguntarle: ¿Juan Carlos cual es la visión de la empresa y misión? y Juan Carlos responde: "Yo solo quiero ganar dinero". Los hermanos mueven la cabeza y dicen creo que primero debimos enfocarnos en esto en vez de invertir en publicidad y marketing y hay que analizar los ratios necesarios que necesitamos, pero bueno el año no se ha perdido".

Justo llega su asistente y dice: "Jefes las ventas han aumentado solamente un $10 \%$ con relación al año anterior y eso que solamente estamos a mitad de año". Juan Carlos, le dice a sus hermanos: "Ya ven, las ventas están bien entonces vamos para adelante", Carlos y Francisco le dicen: "Bueno pero igual hay que ver los indicadores que necesitamos". El asistente les muestra un archivo Excel y comenta: "Hay clientes nuevos, pero muchos clientes antiguos no regresan eso lo he analizado en la mañana, además hay mucho gasto en capacitación por parte de la empresa pues el curso in house de la empresa CURSOS subió sus honorarios en $25 \%$ con relación al año anterior, además veo que el nivel de deuda está aumentando". Ya vez hermano, el asistente nos da la razón analicemos que necesitamos ahorita, porque podemos quebrar muy pronto. Juan Carlos se agarra la cabeza y dice:" Pero las ventas están bien".

Juan Carlos se levanta, se va camino a su carro y se acuerda que cuando caminaba a comprar el local en la revista Mecánica Automotriz que siempre revisaba vio que la empresa Automotriz los Magníficos del Volante había incrementado sus ventas en $30 \%$ cada año, que la participación de mercado aumentó en $10 \%$ al termino del segundo año de operación. Y se preguntaba: "¿será esos los datos que hablan mis hermanos?, pero las ventas están bien, además mucho tiempo vamos a perder analizando y construyendo indicadores eso lo tengo en mi cabeza".

\section{RESUMEN}

El presente caso nos muestra la situación de la empresa Automotriz Chachi Car la cual con ayuda de los hermanos de Juan Carlos buscan ser los referentes y seguir agregando valor en el mercado automotriz. Al inicio de la empresa buscaban solamente incrementar sus ventas, pero después analizan que deben de conocer algunos 
indicadores de gestión como financieros, de operaciones, entre otros y buscan convencer a Juan Carlos la importancia de los indicadores de gestión y su posterior aplicación.

\section{OBJETIVOS DEL CASO}

El presente caso de negocios busca que el docente pueda cumplir los siguientes objetivos de aprendizaje:

- Dar a conocer al alumno los diferentes indicadores de gestión

- Poder implementar un Balanced Score Card en la organización

- Describir el estilo de liderazgo del dueño de la empresa según el liderazgo situacional

\section{DESARROLLO DE CASO}

Se recomienda la lectura previa del caso en forma individual y de ahí puedan contestar las preguntas según el desarrollo de la clase en gorma grupal. Para desarrollar este caso se debe de tener en consideración el siguiente plan de clase.

\section{Introducción (20 minutos)}

El docente debe de preguntar cuáles son los personajes principales del caso, sus competencias, experiencias previas, así como también que estilos de liderazgo tienen los personajes del caso teniendo en consideración el liderazgo situacional.

\section{Identificación de fortalezas de la organización y debilidades (20 minutos)}

El docente debe de buscar que los estudiantes puedan identificar en una matriz FODA donde se muestre las fortalezas, oportunidades, debilidades y amenazas de la organización y su posterior aprovechamiento de las oportunidades en beneficio de la organización

\section{Desarrollo del Balanced Score Card (50 minutos)}

El docente en forma conjunta con los alumnos debe de empezar a diseñar el Balanced Score Card de la organización, empezando por la visión, misión, valores de la empresa.

Tener en consideración las diversas perspectivas como la perspectiva financiera, del cliente, procesos internos y aprendizaje $\mathrm{y}$ crecimiento.

\section{REFERENCIAS}

Gong, Y., Huang, J.-C., \& Farh, J.-L. (2009). Employee Learning Orientation, Transformational Leadership, and Employee Creativity: The Mediating Role of Employee Creative Self-Efficacy. Academy of Management Journal, 52(4), 765-778. doi:10.5465/amj.2009.43670890

Kaplan, R. y Norton, D (1992) The balanced scorecard--measures that drive performance. Harvard Business Review, 70 (1), pp. 71-79

Flores, D., y Rivas, R.(2012). ¿Control de gestión o gestión de control? Contabilidad $y$ Negocios, 7(14),69-80.https://search-proquestcom.ezproxybib.pucp.edu.pe/docview/13189356 49 ? accountid $=28391$

Thompson, G., \& Vecchio, R. P. (2009). Situational leadership theory: A test of three versions. The Leadership Quarterly, 20(5), 837848. doi:10.1016/j.leaqua.2009.06.014

Vega V. y Jácome D. (2019). El balanced scorecard como herramienta de gestión organizacional. Revista Científica Ecociencia, 6(2) https://search-proquestcom.ezproxybib.pucp.edu.pe/docview/22292806 53 ? accountid $=28391$ 